Hamostaseologie 2019; 39(S 01): S1-S92

DOI: $10.1055 / \mathrm{s}-0039-1680209$ Add to LibraryGet PDF

Poster

P07 Paediatric and Neonatal Thrombosis and Haemostasis and Women Issues in Thrombosis and Haemostasis

Georg Thieme Verlag KG Stuttgart · New York

\title{
Recurrent Pediatric Stroke: The Role of Thrombophilia in a Large International Pediatric Stroke Population
}

G. deVeber, F. Kirkham, L.R. Brandao, R. Sträter, G. Kenet, M. Kausch, M. Stoll, A. Torge, V. Ganesan, M. Prengler, U. Nowak-Göttl

$>$ Author Affiliations

Further Information

- $\quad$ Congress Abstract

- $\quad$ Full Text

Objective: Risk factors for arterial ischemic stroke (AIS) in children are multiple and include cardiac disease, vasculopathy, and prothrombotic risk factors (PR). The relevance of these factors to a second AIS event is incompletely understood.

Methods: We conducted a multicenter cohort study to assess the rate of symptomatic stroke recurrence following initial AIS, pooling data on recurrent AIS from the databases held in Canada, Germany, and UK. We followed 894 patients aged 1 month to 18 years (median 6 years) at initial AIS for median 35 months.

Results: 160 of 894 patients (17.9\%) had recurrence from 1 day to 136 months (median 3.1 months) after first AIS. Recurrence was significantly more common in children with (hazard ratio (HR) 2.5, 95\% confidence intervals (CI) 1.92-3.5, p < 0.001) compared to children without vasculopathy. After adjusting for vasculopathy, antithrombin deficiency, elevated lipoprotein (a), and the presence of any combined PR were independently associated with recurrence. Recurrence rates calculated per 100 person-years were 10 (95\%CI: 3-24) for antithrombin deficiency, 6 (95\%CI 4-9) for elevated Lp(a), and 13 (95\%CI 7-20) for combined PR.

Conclusions: Identifying children at increased for recurrent AIS events is important in intensifying preventative measures. Among 894 Canadian, English and German pediatric stroke patients, $17.9 \%$ experienced recurrent AIS at a median of 3.1 months after the index stroke. The presence of more than one prothrombotic risk factor is associated with AIS recurrence in children 